\title{
The clinical course of symptomatic deep vein thrombosis after 3 months of anticoagulant therapy using fondaparinux/edoxaban or fondaparinux/ vitamin $\mathrm{K}$ antagonist
}

This article was published in the following Dove Press journal:

Therapeutics and Clinical Risk Management

\section{Kazuhiro Shimizu \\ Takuo liduka \\ Shuji Sato \\ Hajime Kiyokawa \\ Takahiro Nakagami \\ Hiroshi Mikamo \\ Masayo Kawazoe \\ Mao Takahashi \\ Mahito Noro}

Department of Internal Medicine, Toho University Sakura Medical

Center, Sakura City, Chiba, Japan
Correspondence: Kazuhiro Shimizu Department of Internal Medicine, Toho University Sakura Medical Center, 56I-4 Shimoshizu, Sakura City, Chiba 285-874I, Japan

Tel +8 I 4346288 I I

Fax +81434628820

Email k432@sakura.med.toho-u.ac.jp
Background: For the management of venous thromboembolism (VTE), providing anticoagulant therapy within the therapeutic range has been a major challenge, as conventional therapy with unfractionated heparin (UFH) and vitamin $\mathrm{K}$ antagonist (VKA) requires frequent laboratory monitoring and dose adjustment. Recently, fondaparinux and edoxaban are being used as beneficial alternatives to UFH and VKA.

Methods: We evaluated the clinical course of symptomatic deep vein thrombosis (DVT) in patients who received the 3-month anticoagulation therapy with fondaparinux/edoxaban (Group A; $\mathrm{n}=40$ ) in comparison with the findings from our previous experience of patients who received the fondaparinux/VKA combination (Group B; $\mathrm{n}=33$ ).

Results: In both Groups A and B, serum D-dimer was significantly improved after treatment $(p<0.001)$. The thrombus volume assessed by quantitative ultrasound thrombosis (QUT) score was significantly reduced in both groups $(p<0.001)$. There was no difference in the proportion of patients who were normalized (ie, disappearance of DVT) between the groups, although Group A had significantly more patients who were normalized or improved (ie, disappearance and reduction of DVT) $(p<0.001)$. No bleeding event was observed in either group. However, in one patient in Group B, worsening of DVT and development of symptomatic PE were observed.

Conclusion: Fondaparinux/edoxaban therapy is as effective as fondaparinux/VKA. This treatment has the possible advantage in thrombus regression. This would be a beneficial therapeutic option for both patients and physicians.

Keywords: venous thromboembolism, deep vein thrombosis, anticoagulant therapy, quantitative ultrasound thrombosis score, FXa inhibitors

\section{Introduction}

Venous thromboembolism (VTE), which includes deep vein thrombosis (DVT) and pulmonary embolism (PE), is associated with a variety of clinical situations. In the short term, VTE has the clinically important problem of fatal PE at the acute phase. In the long term, VTE is associated with serious complications, including post-thrombotic syndrome and chronic pulmonary hypertension. Therefore, the most important factor in VTE management is to administer the optimal anticoagulant therapy within the therapeutic range.

Conventional bridging therapy with unfractionated or low-molecular weight heparin (LMWH) followed by vitamin $\mathrm{K}$ antagonist (VKA), such as warfarin, is widely used for treating VTE. However, bridging therapy with unfractionated heparin 
(UFH) and VKA requires frequent laboratory monitoring and dose adjustment, which requires patient compliance and it is often difficult to carry out even for specialist physicians. ${ }^{1}$ Observational studies have demonstrated that optimal management of UFH is difficult to achieve in routine clinical practice. ${ }^{2}$ In addition, UFH poses an 8-10-fold higher risk of heparin-induced thrombocytopenia compared to LMWH. ${ }^{3}$ Thus, fixed dose LMWH has largely replaced UFH as the treatment for VTE.

In Japan, fondaparinux was approved in 2011. The approval of fondaparinux injections for VTE has relieved patients and physicians of the need for complex dose adjustments, 24-h infusion control, and blood tests every 6 h. $^{4}$ In the Hokusai-VTE Study, edoxaban which is an oral direct factor Xa (FXa) inhibitor was used in VTE management. ${ }^{5}$ The European Society of Cardiology (ESC) guidelines recommend the use of edoxaban following acute-phase parenteral anticoagulation therapy. Fondaparinux/edoxaban therapy could become a new option for DVT treatment like UFH/ edoxaban and LMWH/edoxaban.

We undertook the current study in an attempt to evaluate the clinical course of symptomatic DVT after 3 months of fixed-dose anticoagulant therapy.

\section{Methods}

This study was carried out as a single-center, prospective observational study; the study design is shown in Figure 1. The patients were all symptomatic consecutive DVT case series with or without PE. Included in this study were 40 DVT patients (Group A) diagnosed with acute symptomatic DVT who received fondaparinux/edoxaban treatment at the Department of VTE Management Division, Toho University Sakura Medical Center between November 2014 and June 2016.

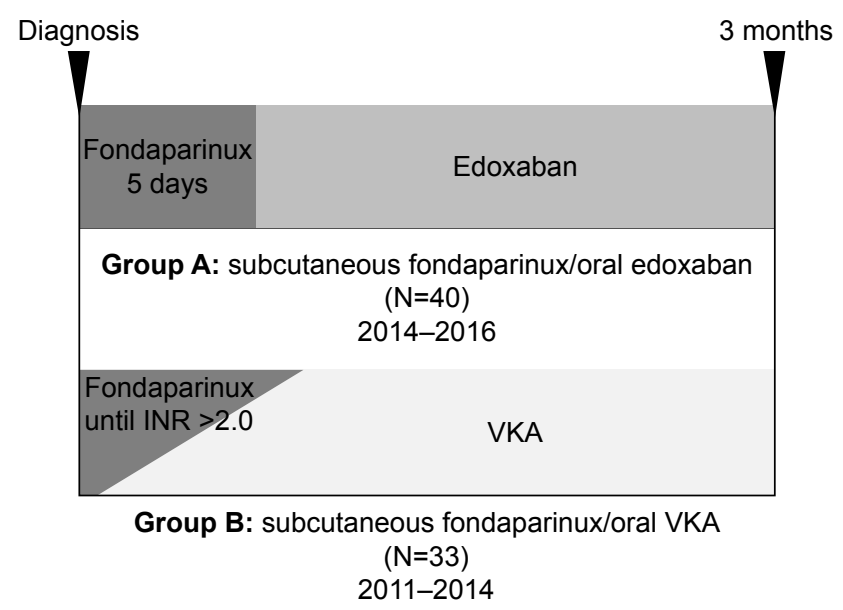

Figure I Study design. The therapy for group A was fondaparinux/edoxaban and group $B$, fondaparinux/VKA.

Abbreviations: INR, international normalized ratio; VKA, vitamin $\mathrm{K}$ antagonist.
Patients gave their informed consent for study participation. DVT was diagnosed based on venous ultrasonography and helical CT scan. Patients were excluded if they would not be available for the 3-month follow-up. All patients were followed up for at least 3 months. During each visit, any signs or symptoms suggesting VTE recurrences or bleeding complications were recorded. In the fondaparinux/edoxaban group (Group A), fondaparinux $7.5 \mathrm{mg}$ was administered by subcutaneous injection once daily for 5 days (to adjust for weight differences, in place of $7.5 \mathrm{mg}, 5 \mathrm{mg}$ was given for body weight below $50 \mathrm{~kg}$ and $10 \mathrm{mg}$ for body weight above $100 \mathrm{~kg}$ ). From day 6, oral edoxaban was given at a dose of $60 \mathrm{mg}$ once daily for the remainder of the treatment period. A reduced edoxaban dose was used for patients with moderate renal impairment, creatinine clearance $(\mathrm{CrCl}) 30-50 \mathrm{~mL} / \mathrm{min}$, or for those with a body weight of $\leq 60 \mathrm{~kg}$ who were receiving P-glycoprotein inhibitors. We excluded the patients with $\mathrm{CrCl}<30$. A total of 24 patients in group A were started with $30 \mathrm{mg}$ edoxaban because of their body weight being $<60 \mathrm{~kg}$. All participants in this study were adult patients.

To allow for a quantitative assessment of the venous ultrasound, the residual diameters of the common femoral, superficial femoral and popliteal veins were measured under full compression. And we also assessed the DVT 3 months after the treatment by ultrasonography. We compared the findings with baseline and evaluated the results as normalized, improved (ie, a decrease of $4 \mathrm{~mm}$ or more), unchanged, or deteriorated (ie, an increase of $4 \mathrm{~mm}$ or more).${ }^{6,7}$ In addition, the DVT thrombus volume was calculated by quantitative ultrasound thrombosis (QUT) score, a scoring system which was recently developed at Toho University. ${ }^{8,9}$

\section{Measurement of the QUT score}

We evaluated the DVT thrombus volume using the QUT score. In the QUT scoring system, the deep veins are separated into several segments: inferior vena cava, right pelvic vein, left pelvic vein, right femoral vein, left femoral vein, right below-knee veins, and left below-knee veins. When there are multiple below-knee vein thrombosis, each are evaluated. Two points are given for an occlusion by a thrombus, and one point for the presence of a thrombus but no occlusion. The sum of the points in each segment is then evaluated. ${ }^{8,9}$

Expert physiology laboratory technicians performed the ultrasonography using Aplio XG (Toshiba Medical System, Tochigi, Japan) with a linear probe.

\section{Statistical analysis}

All data were expressed as mean \pm standard deviation. Unpaired Student's $t$-test was used to compare the baseline 
characteristics between the fondaparinux/edoxaban group (Group A) and the fondaparinux/warfarin group (Group B). The comparison of thrombotic changes between the two groups was analyzed by an unpaired $t$-test. A paired $t$-test was used to compare the mean values before and after treatment. Statistically significant differences were considered at $p<0.05$. Statistical analyses were performed using the statistical package SPSS Version 22.0 (IBM, Chicago, IL, USA). We performed the post hoc power analysis using G*Power 3 software (Dusseldorf, Germany) between group $\mathrm{A}$ and $\mathrm{B}$ about the regression of thrombus. ${ }^{10}$

\section{Ethics}

Participants gave written informed consent after a detailed description of the procedures, in accordance with the Declaration of Helsinki, and the study protocol was approved by the ethics committee of Toho University Sakura Medical Center (approved NO 2015-024) and (UMIN-CTR ID: UMIN000018515).

\section{Results}

As a control group (Group B), data from 33 DVT patients who had received treatment with fondaparinux plus warfarin for 3 months at our hospital between 2011 and 2014 and had undergone outcome evaluation at the treatment completion were used. Since the time of the approval of fondaparinux, the combination of fondaparinux and warfarin has been used as the first-choice treatment for DVT in our hospital. In that regimen, fondaparinux was continued for at least 5 days of overlap and until an international normalized ratio (INR) $\geq 2$ was achieved. Through the 3 -month treatment period, time in therapeutic range (TTR) was $62.2 \%$. The mean \pm SD of overlapping use of fondaparinux was $8.5 \pm 2.2$ days with a $95 \%$ confidence interval of 7.7-9.2. Japanese Circulation Society guidelines recommend a target INR range of 1.5-2.5. ${ }^{11}$

The patients' characteristics are listed in Table 1. There were no differences between the two groups in age, sex, height, body weight, body mass index, the location of DVT, obesity, steroids' use, hormonal contraceptives, current smoking, COPD, infection, recent surgery, cancer, chemotherapy, fragility, diabetes mellitus (DM) and hypertension (HT). Further, there were no differences between serum albumin, creatinine and D-dimer levels. Group B had more patients with complications of pulmonary embolism and dyslipidemia.

The changes in laboratory markers are listed in Table 2. In Group A, there were significant increases from baseline in the serum albumin $(p<0.001)$ and creatinine levels $(p<0.05)$ at the completion of the 3-month treatment. In both Groups $\mathrm{A}$
Table I Patient characteristics

\begin{tabular}{|c|c|c|c|}
\hline Variables & $\begin{array}{l}\text { Group A } \\
\text { fondaparinux/ } \\
\text { edoxaban } \\
(\mathbf{N}=40)\end{array}$ & $\begin{array}{l}\text { Group B } \\
\text { fondaparinux/ } \\
\text { VKA } \\
(\mathbf{N}=33)\end{array}$ & $p$-value \\
\hline Age (years) & $67.6( \pm 16.3)$ & $63.4( \pm 17.5)$ & n.s. \\
\hline Male (\%) & $19(47.5 \%)$ & $16(48.5 \%)$ & n.s. \\
\hline Height (cm) & 159.8 (土9.8) & $161.3( \pm 9.8)$ & n.s. \\
\hline Body weight $(\mathrm{kg})$ & $60.7( \pm 14.6)$ & $66.2( \pm 13.9)$ & n.s. \\
\hline Body mass index $\left(\mathrm{kg} / \mathrm{m}^{2}\right)$ & $23.6( \pm 4.0)$ & $25.1( \pm 3.4)$ & n.s. \\
\hline $\begin{array}{l}\text { Proximal from } \mathrm{FV} \text { at } \\
\text { right side }\end{array}$ & $9(22.5 \%)$ & $8(24.2 \%)$ & n.s. \\
\hline $\begin{array}{l}\text { Proximal from FV at } \\
\text { left side }\end{array}$ & $18(45.0 \%)$ & $20(60.6 \%)$ & n.s. \\
\hline PV at right side & $10(25.0 \%)$ & $10(30.3 \%)$ & n.s. \\
\hline PV at left side & I 8 (45.0\%) & I 4 (42.4\%) & n.s. \\
\hline Distal DVT at right side & 18 (45.0\%) & $19(57.6 \%)$ & n.s. \\
\hline Distal DVT at left side & $26(65.0 \%)$ & 15 (45.5\%) & n.s. \\
\hline Pulmonary embolism & $16(40.0 \%)$ & $22(66.7 \%)$ & $p=0.023$ \\
\hline BMI $\left(\mathrm{kg} / \mathrm{m}^{2}\right)>25$ & I I (27.5\%) & $14(42.4 \%)$ & n.s. \\
\hline BMI $\left(\mathrm{kg} / \mathrm{m}^{2}\right)>30$ & $2(5.0 \%)$ & $4(12.1 \%)$ & n.s. \\
\hline Steroids user & $6(15.0 \%)$ & $4(12.1 \%)$ & n.s. \\
\hline Hormonal contraceptives & I (2.5\%) & I (3\%) & n.s. \\
\hline Current smoking & $3(7.5 \%)$ & 7 (2I.2\%) & n.s. \\
\hline COPD & $4(10.0 \%)$ & $3(9.1 \%)$ & n.s. \\
\hline Infection & $5(12.5 \%)$ & $2(6.1 \%)$ & n.s. \\
\hline Recent surgery & $8(20.0 \%)$ & $4(12.1 \%)$ & n.s. \\
\hline Cancer & $8(20.0 \%)$ & $8(24.2 \%)$ & n.s. \\
\hline Chemotherapy & $3(7.5 \%)$ & $3(9.1 \%)$ & n.s. \\
\hline Fragility* & $19(47.5 \%)$ & $13(39.4 \%)$ & n.s. \\
\hline DM & 7 (I7.5\%) & I I (33.3\%) & n.s. \\
\hline HT & 14 (35.0\%) & $12(36.4 \%)$ & n.s. \\
\hline DL & I I (27.5\%) & 19 (57.6\%) & $p=0.010$ \\
\hline Albumin & $3.9( \pm 0.6)$ & $4.2( \pm 0.6)$ & n.s. \\
\hline Creatinine & $0.78( \pm 0.23)$ & $0.83( \pm 0.23)$ & n.s. \\
\hline D-dimer & $14.0( \pm \mid 2.8)$ & I3.I ( \pm 10.9$)$ & n.s. \\
\hline
\end{tabular}

Notes: Values are mean ( \pm standard deviation) or frequency (\%). *Fragility was defined as one or more of the following criteria: calculated creatinine clearance (CCR) $<50 \mathrm{~mL} / \mathrm{min}, \mathrm{BW}<50 \mathrm{~kg}$ or age $>75$ years.

Abbreviations: VKA, vitamin K antagonist; FV, femoral vein; PV, popliteal vein; DVT, deep vein thrombosis; BMI, body mass index; COPD, chronic obstructive pulmonary disease; DM, diabetes mellitus; DL, dyslipidemia; HT, hypertension; n.s., not significant.

and B, the serum D-dimer level was significantly improved $(p<0.001)$.

No bleeding event was observed in either group. In group B, during the treatment period, worsening of DVT and development of symptomatic PE were observed in one patient (female; age 25 years) who was receiving chemotherapy for ovarian cancer. In that patient, PT-INR was 2.08 and D-dimer was $28.72 \mu \mathrm{g} / \mathrm{mL}$ at the last assessment before the onset of the event.

At the completion of the 3-month treatment period, an imaging study was performed to evaluate therapeutic outcomes. The clinical course of DVT after the 3 months of therapy is shown in Figure 2. By appropriate anticoagulant 
Table 2 Changes in laboratory markers

\begin{tabular}{|c|c|c|c|c|c|c|}
\hline \multirow[t]{2}{*}{ Variable } & \multicolumn{3}{|c|}{ Group $A(\mathbf{N}=40)$} & \multicolumn{3}{|c|}{ Group B $(\mathbf{N}=33)$} \\
\hline & Baseline & 3 months & $p$-value & Baseline & 3 months & $p$-value \\
\hline Albumin $(\mathrm{g} / \mathrm{mL})$ & $3.9( \pm 0.6)$ & $4.3( \pm 0.3)$ & $p<0.001$ & $4.2( \pm 0.6)$ & $4.3( \pm 0.3)$ & n.s. \\
\hline Creatinine (mg/dL) & $0.78( \pm 0.23)$ & $0.82( \pm 0.26)$ & $p<0.05$ & $0.83( \pm 0.23)$ & $0.80( \pm 0.21)$ & n.s. \\
\hline D-dimer $(\mu g / m L)$ & $14.0( \pm \mid 2.8)$ & $\mathrm{I} . \mathrm{I}( \pm \mathrm{I} .2)$ & $p<0.001$ & $13.1( \pm 10.9)$ & $0.9( \pm I .0)$ & $p<0.00$ I \\
\hline
\end{tabular}

Note: Values are mean ( \pm standard deviation).

Abbreviation: n.s., not significant.

therapy, the thrombus in the lower limbs regressed in each group, respectively. There was no difference in the proportion of patients who were normalized (ie, disappearance of DVT) between the groups. Group A had significantly more patients who were normalized or improved (ie, disappearance and reduction of DVT) $(p<0.001)$.

The evaluation for the volume of thrombus is shown in Figure 3. The thrombus volume as assessed by QUT score was significantly reduced in both groups after the completion of the 3-month treatment period $(p<0.001)$; the mean $\pm \mathrm{SD}$ scores changed from $5.2 \pm 2.5$ to $1.3 \pm 1.3$ in group A and from $5.0 \pm 2.8$ to $2.6 \pm 2.4$ in group B.

The comparison of the change in QUT score ( $\triangle$ QUT Score) is shown in Figure 4. There was a significantly greater improvement of thrombosis in Group A than Group B $(-3.8 \pm 2.4$ vs $-2.4 \pm 2.7 ; p<0.05)$. The detection power was $68 \%$ ( $p<0.05$, two tails) in this study.

\section{Discussion}

Based on evidence from clinical trials, the American College of Chest Physicians' (ACCP) guidelines recommend 3 months of anticoagulant therapy in patients with VTE

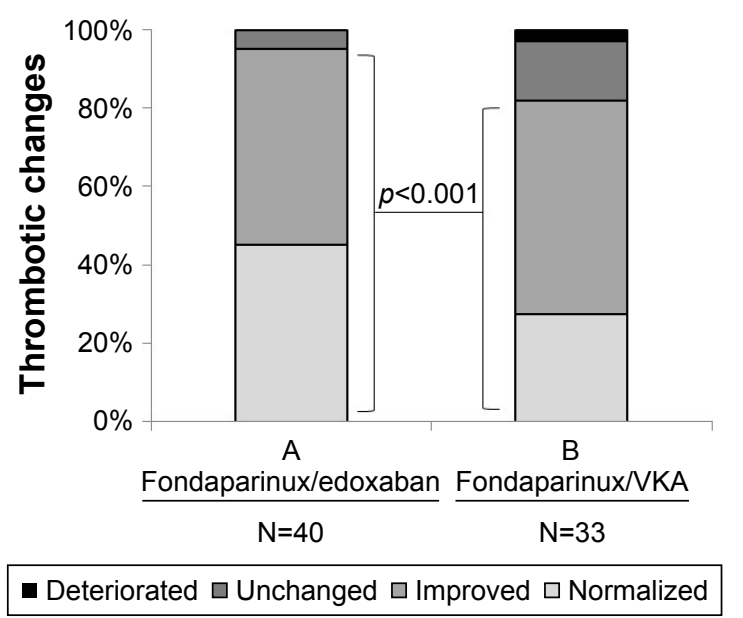

Figure 2 The comparison of thrombotic changes before and after treatment between groups $A$ and $B$.

Abbreviation: VKA, vitamin $\mathrm{K}$ antagonist. secondary to recent surgery or to a nonsurgical transient risk factor, and extended treatment duration in those with active cancer or unprovoked VTE. ${ }^{12}$ The goal of therapy for DVT is to prevent the extension of thrombus, acute $\mathrm{PE}$, recurrence of thrombosis, and the development of late complications such as pulmonary hypertension and postthrombotic syndromes.

Kearon and Akl reported that it takes about 3 months to complete "active treatment" of VTE, with further treatment serving to prevent new episodes of thrombosis ("pure secondary prevention"). The goal of active treatment is to suppress the acute episode of thrombosis, whereas the aim of subsequent anticoagulation is to prevent new episodes of VTE that are unrelated to the index event. ${ }^{13}$ In this study, our aim was to investigate the clinical course of symptomatic DVT after "active treatment." Of course, many patients continued with further treatment. For that reason, we evaluated DVT after 3 months anticoagulation therapy.

Conventional treatment starts with a rapidly acting parenteral anticoagulant, usually LMWH in Western countries and UFH in Japan, which is followed by overlapping use with VKA, such as warfarin. The parenteral anticoagulant is given for at least 5 days and is stopped when a therapeutic response to VKA has been achieved as evidenced by an INR of between 2 and 3. VKA is then continued as long-term therapy for a minimum of 3 months. At this point, the decision to stop or continue treatment depends upon the balance between the risk of recurrence if VKA is stopped and the risk of bleeding if it is continued.

While experts recommend that patients spend at least $65 \%$ of their TTR, this is seldom achieved or sustained over time. Patients are more likely to have INR below the therapeutic range, exposing them to significant risk of adverse clinical events. ${ }^{14}$ In the Hokusai VTE Study, among patients receiving warfarin, the INR was in the therapeutic range for $63.5 \%$ of the time, above 3.0 for $17.6 \%$ of the time, and below 2.0 for $18.9 \%$ of the time. ${ }^{5}$ In the Einstein DVT Study, the INR was in the therapeutic range $2.0-3.0$ for $57.7 \%$ of the time, above 3.0 for $16.2 \%$ of the time, and below 2.0 

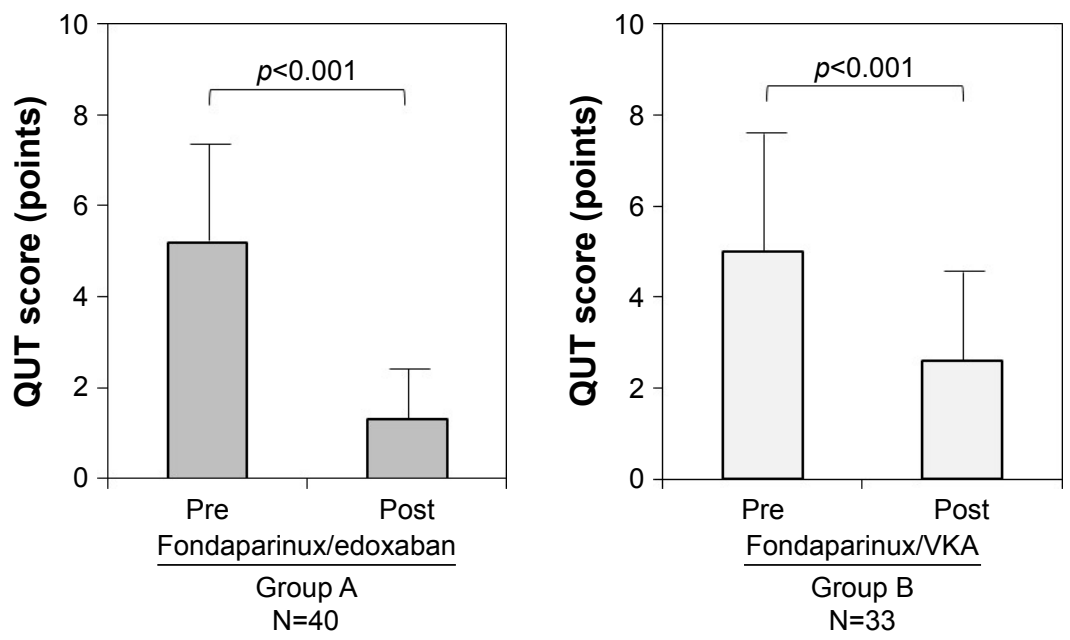

Figure 3 QUT score in patients with DVT. A statistically significant decrease was observed after treatment in both groups. Abbreviations: QUT, quantitative ultrasound thrombosis; VKA, vitamin K antagonist; DVT, deep vein thrombosis.

for $24.4 \%$ of the time. ${ }^{15}$ Mean time in the therapeutic range observed in recent VTE randomized controlled trials were as follows, AMPLIFY VTE 61\%, EINSTEIN-DVT 58\%, EINSTEIN-PE 63\% and Hokusai-VTE 64\%. It is hard to keep a high TTR control level in a clinical trial. We also found it more difficult to keep a high TTR control level in daily practice. Maintaining the antithrombotic therapy within the therapeutic range was a major challenge for a long time before the introduction of fondaparinux and edoxaban as alternatives to UFH and VKA, respectively.

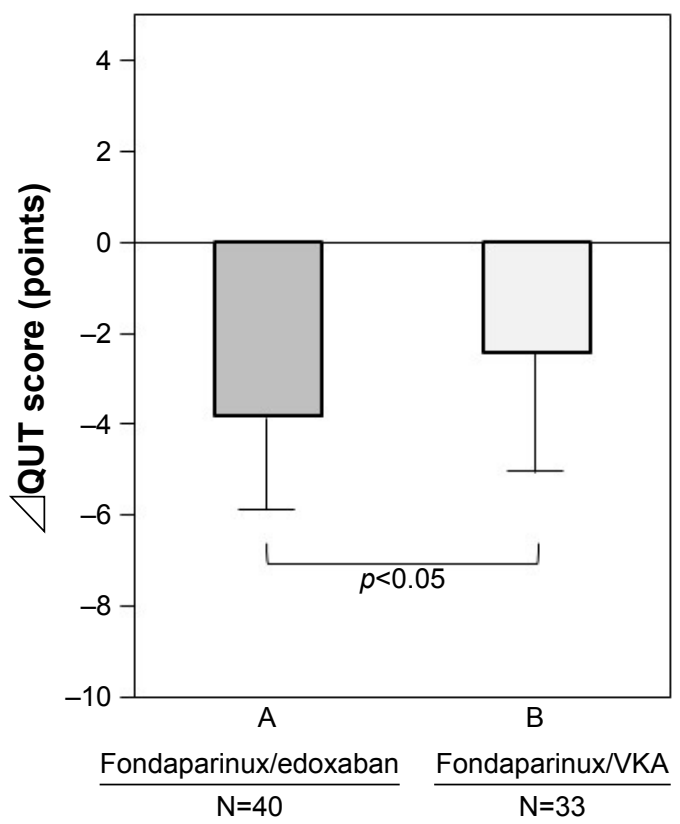

Figure 4 The comparison of the $\triangle$ QUT score between groups A and B. Group A was statistically improved compared to group B.

Abbreviations: $\triangle \mathrm{QUT}$, change in the quantitative ultrasound thrombosis; VKA, vitamin $\mathrm{K}$ antagonist.
These have more easily facilitated the achievement of optimal antithrombotic therapy. In Japan, the open-label, randomized J-EINSTEIN study reported that rivaroxaban reduced DVT significantly more than the control treatment with UFH and warfarin. ${ }^{16}$

Venous ultrasonography has become the most widely used diagnostic modality for the diagnosis and exclusion of acute DVT. Duplex ultrasound is considered to be the primary noninvasive diagnostic method for DVT. The accuracy of venous ultrasonography in comparison to venography has been well established. The weighted mean sensitivity and specificity of venous ultrasonography (including all types) for the diagnosis of symptomatic proximal DVT are $97 \%$ and $94 \%$, respectively. ${ }^{17}$ In addition, Hisatake et al have developed a new scoring system (ie, QUT score) for the evaluation of DVT, which enables the assessment of thrombosis in each lesion. ${ }^{8,9}$ In the present study, we used this scoring system to evaluate the clinical course of DVT.

As a main objective, we compared the effects of two therapeutic options for DVT, both of which were used as a standard regimen at the time in our VTE specialist outpatient clinic division. The combination of fondaparinux and warfarin was useful in terms of use of the fixed dose, however, based on our experience, it required an overlapping treatment period for an average of 8.5 days to attain the effect of warfarin to within the therapeutic range on admission. In 2014, edoxaban became available as an alternative option for VKA and consequently, more stable management of DVT became possible.

In the process of thrombus formation, two main pathways, blood coagulation and platelet aggregation, play 
pivotal roles. Selective FXa inhibitors do not directly inhibit platelet aggregation mediated by thrombin, ${ }^{18}$ but by inhibiting FXa, FXa inhibitors are expected to suppress thrombin generation and thus they have the potential to indirectly decrease thrombin-mediated platelet aggregation. Honda et al previously compared the antithrombotic effects of edoxaban and fondaparinux in rat venous and arterial thrombosis models. ${ }^{19,20}$ These two selective FXa inhibitors significantly and completely prevented venous thrombus formation in a prophylaxis study (Fukuda et al) ${ }^{19}$ and significantly regressed venous thrombi in a treatment study (Morishima et al). ${ }^{20}$

In the present study, a small increase in the creatinine level was observed in Group A $(p<0.05)$. This may be associated with renal excretion or incidental change.

Owing to the introduction of FXa inhibitors that provide stable antithrombotic effects as well as the development of the new quantitative assessment tool, QUT score, we confirmed the effects of the fondaparinux plus edoxaban therapy on DVT as compared with the fondaparinux plus VKA therapy in accordance with the recommendation of the guidelines (minimum 3-month treatment).

\section{Study limitations}

Firstly, this study was a single-center observational study of fondaparinux/edoxaban in 40 adults with DVT compared with a historical cohort of 33 adults using fondaparinux/ VKA. For that reason, sample size was very limited. The Hokusai-VTE study which included 4,921 DVT patients and 3,319 pulmonary thromboembolism patients showed that edoxaban administered once daily after initial heparin was not inferior to standard therapy with warfarin after initial heparin, with significantly less bleeding. In our study, we tried to evaluate the clinical course of symptomatic DVT after 3 months of anticoagulation therapy from the point of thrombus regression. We performed the post hoc power analysis using $\mathrm{G}^{*}$ Power 3 software between group $\mathrm{A}$ and $\mathrm{B}$ about the regression of thrombus. The detection power was $68 \%(p<0.05$, two tails $)$ in this study. The detection power was slightly weak, but we could not increase the sample size because of the comparison with a past group.

Secondly, the study patients were all symptomatic DVT. The patient characteristics of both groups were similar as shown in Table 1. The rate of PE was significantly higher in group B (fondaparinux/edoxaban group vs fondaparinux/VKA was $40 \%$ to $66.7 \%, p<0.05$ ). But this study was to investigate the clinical course of DVT and not the rate of PE, so we thought that this had little effect on the overall results.

\section{Conclusion}

Providing anticoagulant therapy within the therapeutic range stabilized many DVTs in the 3 months observation. Fondaparinux/edoxaban therapy is as effective as fondaparinux/VKA. This treatment has the possible advantage in thrombus regression. This would be a beneficial therapeutic option for both patients and physicians.

\section{Acknowledgments}

The authors thank Takeshi Sasaki, Tsuyoshi Tabata, Osamu Takahashi and Norio Kon for their technical assistance in the ultrasonography.

The authors deeply appreciate Professor Takanobu Tomaru, Professor Hirofumi Noike, Shunsuke Todani, Takurou Itou, Masahiro Iwakawa, and Yuta Sugizaki for their management of the medical environment.

\section{Disclosure}

K Shimizu has received honoraria for oral presentations from Bayer Yakuhin and Daiichi Sankyo. The other authors report no conflicts of interest in this work.

\section{References}

1. Goldhaber SZ, Bounameaux H. Pulmonary embolism and deep vein thrombosis. Lancet. 2012;379(9828):1835-1846.

2. Hylek EM, Regan S, Henault LE, et al. Challenges to the effective use of unfractionated heparin in the hospitalized management of acute thrombosis. Arch Intern Med. 2003;163(5):621-627.

3. Martel N, Lee J, Wells PS. Risk for heparin-induced thrombocytopenia with unfractionated and low-molecular-weight heparin thromboprophylaxis: a meta-analysis. Blood. 2005;106(8):2710-2715.

4. Büller HR, Davidson BL, Decousus H, et al. Subcutaneous fondaparinux versus intravenous unfractionated heparin in the initial treatment of pulmonary embolism. N Engl J Med. 2003;349(18):1695-1702.

5. Hokusai-VTE Investigators, Büller HR, Décousus H, Grosso MA, et al. Edoxaban versus warfarin for the treatment of symptomatic venous thromboembolism. N Engl J Med. 2013;369:1406-1415.

6. Prandoni P, Lensing AWA, Prins MH, et al. Residual venous thrombosis as a predictive factor of recurrent venous thromboembolism. Ann Intern Med. 2002;137(12):955-960.

7. Prandoni $\mathrm{P}$, Lensing AWA, Bernardi E, et al. The diagnostic value of compression ultrasonography in patients with suspected recurrent deep vein thrombosis. Thromb Haemost. 2002;88(3):402-406.

8. Hisatake S, Kabuki T, Kiuchi S, et al. Short-term subcutaneous fondaparinux and oral edoxaban for acute venous thromboembolism. Circ J. 2017;81(6):855-861.

9. Kabuki T, Nakanishi R, Hisatake S, et al. A treatment strategy using subcutaneous fondaparinux followed by oral rivaroxaban is effective for treating acute venous thromboembolism. J Cardiol. 2017;70(2): 163-168.

10. Faul F, Erdfelder E, Lang AG, Buchner A. G*Power 3: a flexible statistical power analysis program for the social, behavioral, and biomedical sciences. Behav Res Meth. 2007;39(2):175-191. 
11. JCS Joint Working Group. Guidelines for the diagnosis, treatment and prevention of pulmonary thromboembolism (JCS2009). Japanese Circulation Society. Circ J. 2011;75:1258-1281.

12. Kearon C, Akl EA, Comerota AJ, et al. Antithrombotic therapy for VTE disease: Antithrombotic Therapy and Prevention of Thrombosis, 9th ed: American College of Chest Physicians Evidence-Based Clinical Practice Guidelines. Chest. 2012;141(2 Suppl):e419S-e496S.

13. Kearon C, Akl EA. Duration of anticoagulant therapy for deep vein thrombosis and pulmonary embolism. Blood. 2014;123(12):1794-1801.

14. Schein JR, White CM, Nelson WW, Kluger J, Mearns ES, Coleman CI. Vitamin K antagonist use: evidence of the difficulty of achieving and maintaining target INR range and subsequent consequences. Thromb J. 2016;14:14.

15. EINSTEIN Investigators. Oral rivaroxaban for symptomatic venous thromboembolism. N Engl J Med. 2010;363:2499-2510.
16. Yamada N, Hirayama A, Maeda H, et al. Oral rivaroxaban for Japanese patients with symptomatic venous thromboembolism - the J-EINSTEIN DVT and PE program. Thromb J. 2015;13:2.

17. Zierler BK. Ultrasonography and diagnosis of venous thromboembolism. Circulation. 2004;109(12 Suppl 1):I9-I14.

18. Furugohri T, Isobe K, Honda Y, et al. DU-176b, a potent and orally active factor Xa inhibitor: in vitro and in vivo pharmacological profiles. J Thromb Haemost. 2008;6(9):1542-1549.

19. Fukuda T, Tsuji N, Honda Y, Kamisato C, Morishima Y, Shibano T. Comparison of antithrombotic efficacy between edoxaban, a direct factor Xa inhibitor, and fondaparinux, an indirect factor Xa inhibitor under low and high shear rates. Thromb Haemost. 2011;106(6):1062-1068.

20. Morishima Y, Kamisato C, Honda Y. Treatment of venous thrombosis with an oral direct factor Xa inhibitor edoxaban by single and multiple administrations in rats. Eur J Pharmacol. 2014;742:15-21.
Therapeutics and Clinical Risk Management

\section{Publish your work in this journal}

Therapeutics and Clinical Risk Management is an international, peerreviewed journal of clinical therapeutics and risk management, focusing on concise rapid reporting of clinical studies in all therapeutic areas, outcomes, safety, and programs for the effective, safe, and sustained use of medicines. This journal is indexed on PubMed Central, CAS,

\section{Dovepress}

EMBase, Scopus and the Elsevier Bibliographic databases. The manuscript management system is completely online and includes a very quick and fair peer-review system, which is all easy to use. Visit http://www.dovepress.com/testimonials.php to read real quotes from published authors.

Submit your manuscript here: http://www.dovepress.com/therapeutics-and-clinical-risk-management-journal 